\title{
Mechanical microenvironments of living cells: a critical frontier in mechanobiology
}

\author{
Guoyou Huang ${ }^{1,2} \cdot$ Feng Xu ${ }^{1,2} \cdot$ Guy M. Genin ${ }^{1,2,3,4} \cdot$ Tian Jian Lu ${ }^{5,6}$
}

Published online: 10 April 2019

(c) The Chinese Society of Theoretical and Applied Mechanics and Springer-Verlag GmbH Germany, part of Springer Nature 2019

The fields of biomechanics and mechanobiology have long been predicated on the premise that mechanics governs cell behavior. However, over the past few years, a growing body of evidence has suggested that the mechanical environment very close to cells-the cell microenvironment-plays the most important role in determining what a cell feels and how it responds to tissue-level stimuli. To complicate matters further, cells can actively manipulate their microenvironments through pathways of recursive mechanobiological feedback. Harnessing this recursive behavior to understand and control cell physiology and pathophysiology is a critical frontier in the field of mechanobiology. Recent results suggest that the key to opening this scientific frontier to investigation and engineering applications is understanding a different frontier: the physical frontier that cells face when probing their mechanical microenvironments.

The mechanical microenvironments of living cells are now understood to play a critical role in regulating a broad range of cell behaviors, from development and healing to degradation and disease. Unraveling and controlling these is a critical need for the biomechanics and mechanobiology community. Engineering heterogeneous and dynamic cell mechanical microenvironments is a major focus of the field.

Guoyou Huang

wwwgyhuang@xjtu.edu.cn

$\triangle$ Feng Xu

fengxu@xjtu.edu.cn

$\triangle$ Guy M. Genin

genin@wustl.edu

$\triangle$ Tian Jian Lu

tjlu@xjtu.edu.cn

1 The Key Laboratory of Biomedical Information Engineering of Ministry of Education, School of Life Science and Technology, Xi' an Jiaotong University, Xi' an 710049, China

2 Bioinspired Engineering and Biomechanics Center (BEBC), Xi' an Jiaotong University, Xi' an 710049, China
Studies on mechanobiology and cell mechanotransduction have greatly enriched our knowledge and provided potential molecular targets for clinical mechanotherapies. This branch of mechanobiology is now becoming a field of its own, and represents an important frontier in theoretical and applied mechanics.

To further the development of this field, the International Union of Theoretical and Applied Mechanics (IUTAM) sponsored the IUTAM Symposium on Mechanical Environments of Living Cells from 28 to 30 June, 2018, in Xi' an, China, along with three major national-level research centers focusing on these issues: the Bioinspired Engineering and Biomechanics Center at Xi' an Jiaotong University, Xi'an, China; the Mechanobiology Institute of the National University of Singapore; and the NSF Science and Technology Center for Engineering MechanoBiology, a joint center between Washington University in St. Louis and the University of Pennsylvania, USA. Major international university alliances supported this meeting as well, including the University Alliance of the Silk Road, based at Xi' an Jiaotong University, and the McDonnell International Scholars Academy, based at Washington University in St. Louis. The meeting was additionally sponsored by the US NIH/Interagency

3 Department of Mechanical Engineering and Materials Science, Washington University in St. Louis, St. Louis, MO 63130, USA

$4 \quad$ NSF Science and Technology Center for Engineering Mechanobiology, Washington University in St. Louis, St. Louis, MO 63130, USA

$5 \quad$ State Key Laboratory of Mechanics and Control of Mechanical Structures, Nanjing University of Aeronautics and Astronautics, Nanjing 210016, China

$6 \quad$ State Key Laboratory for Strength and Vibration of Mechanical Structures, Xi'an Jiaotong University, Xi' an 710049, China 
Modeling and Analysis Group's (IMAG's) working group on integrated multiscale biomechanics experiment and modeling (I $\mu$ BEAM), by the National 111 Program of China, and by the National Natural Science Foundation of China.

At the IUTAM meeting, a group of leading international researchers met to discuss the most important questions in this field, including the development of biomimetic and micro/nanoengineered materials for simulating the native cell microenvironments, characterization of cell microenvironments, predictive models for cell mechanobiology, and applications of engineered cell microenvironments. This special thematic issue of Acta Mechanica Sinica brings together 12 articles contributed by leading researchers with the aims of capturing the essence of this important IUTAM meeting, and of helping solidify this emerging and important field. A summary of key observations from this meeting and this issue follows.

\section{The mechanical microenvironment drives development, disease, and decisions}

The mechanical microenvironment is now understood to be a critical factor in directing how the cells of multicellular animals interact with one another and choose their phenotype. Why do cells of multicellular animals have these unusual behaviors, and from where do they arise? The special issue includes an article from Chen and Wang [1], who present an interesting cell stiffness postulate to explain the evolution of eukaryotes from simple cells to complex multicellular organisms. They propose that early soft, unicellular organisms might have evolved the ability to modulate, and especially to stiffen, their cytoskeletons to protect their structural integrity. The premise here is that the phenomenon of stiffness matching in cells, in which cells tend to adapt their stiffness to match that of their matrix in both two-dimensional (2D) and three-dimensional (3D) environments, is needed to prevent or at least partially control damage to cells when the tissues in which they reside are stressed mechanically. In plants and fungi, this is often achieved by a cell wall, but animal cells need to maintain form, change shape, and move. These requirements might be a fundamental underpinning of multicellular animal evolution, and would explain why mechanobiology is a fundamental feature of animal cells, as inbuilt as genome evolution and cadherin evolution in the development of our multicellular ancestors.

These phenomena underlie collective cell behaviors in tissue morphogenesis, wound healing, and cancerous cell invasion. In a review of recent experimental and theoretical studies of collective cell behaviors in 2D and 3D substrates and tissues, He et al. [2] encapsulate how collective cell polarization and orientation are driven by the local stresses that cells within a cell layer or tissue feel. This is termed stress-driven cell polarity. Their findings provide a new perspective for understanding collective cell behaviors in living organisms and suggest new opportunities for treating disease.

At the level of organs, analogous mechanical cues are emerging as key aspects of cell physiology and pathology. In the case of the liver, as summarized by You et al. [3], these mechanical cues have critical biological significance, driving regulatory factors and cellular responses during liver pathogenesis. Although not fully understood, these are especially important in fibrosis and cirrhosis of the liver. Clearly, a deeper understanding of the contributions of mechanical cues to liver homeostasis and pathogenesis is essential for discovering methods for diagnosis and identification of new therapeutic targets for diseases of the liver.

A long-underappreciated mechanosensitive component of cells is the nucleus. The nucleus is now understood to be mechanosensitive. From the perspective of mechanotransduction, the nucleus shows the ability both to read microenvironmental mechanical cues transmitted through the cytoskeleton and to respond to these cues with fundamental nuclear mechanotransduction. An additional layer of this appears in cancer, where metastasis is the leading cause of mortality. Nuclear mechanics is a critical feature in regulating migration of cells through the constricted passageways that exist in 3D tissues. Recent findings on possible mechanisms for cell migration through such constrictions were reviewed by Xia et al. [4]. The authors report that nuclear envelope rupture, DNA damage, and genomic variation all arise during and after constricted migration, and have shown this through both experiment and modeling.

One of the most celebrated examples of how mechanics drives cell function is the role of mechanical cues in driving stem cell differentiation. Stem cells are important both physiologically and scientifically because of their self-renewal capacity and multiple-lineage differentiation potential. Mechanical cues have been found to play important roles in controlling stem cell fate, but the interactions of these with other cues such as biochemical cues are still not clear. Investigating the relationship between the stiffness of 3D demineralized bone scaffolds and the behavior of mesenchymal stem cells (MSCs) under hypoxic conditions, Sun et al. [5] report that scaffolds with low stiffness improve the proliferation and osteogenic differentiation of MSCs under hypoxic conditions. Understanding how mechanical cues interact with other features of the cell microenvironment is critical to harnessing these phenomena clinically, and will certainly reveal potential pathways for improving stem cell therapies. 


\section{Characterization and engineering of the cell microenvironment are ongoing challenges}

A major technical challenge in engineering and understanding the cell microenvironment is the difficulty in developing accurate in situ characterization methods. A very promising approach is that of Wang et al. [6], who introduced a scanning electrochemical microscopy (SECM)-based method to map the surface topography and measure oxygen permeability around hydrogel microwell arrays in aqueous solution. The method not only shows high spatial resolution for in situ characterization of hydrogel surface topography and oxygen permeability, but also shows great promise in detecting other critical species and biomarkers of cell function such as $\mathrm{pH}$ and the presence of reactive oxygen species. A critical direction for this in future will be characterizing live cells in a 3D microenvironment.

Defining, characterizing, and replicating these microenvironments is the critical challenge for engineering $3 \mathrm{D}$ tissue models in vitro. A modified hanging-drop method developed by Gao et al. [7] enables controlled production of 3D cell spheroids. The most important factor here is the balance between the effects of surface tension and those of gravity. $\beta$-TC- 6 cell spheroids with optimized diameters were fabricated, with morphology and functionality very similar to those of primary islets. Technologies such as these with welldefined microenvironments could be useful as engineering tissue models for basic research and drug screening applications.

\section{Integrated multiscale experiment and modeling is a critical frontier}

A major obstacle in characterizing cellular phenomena is the challenge of integrating modeling and experiment. The quantifying measurements of the cell microenvironment that are possible to make are typically indirect, and must be interpreted in the context of a model. Conversely, predictions of models are often not possible to observe directly, and require design of idealized bespoke experimental frameworks for testing.

One of the most fundamental of these challenges is quantifying the volumetric response of isolated cells embedded in a 3D matrix. These volumetric responses are important for regulation of the cell cycle, and may factor into a range of physiological and pathophysiological responses to mechanical loading. However, relating observed volumetric responses to cell mechanics requires a mechanical model. A mathematical model for describing the volumetric response of an ellipsoidal compressible liquid inclusion embedded in a strained 3D matrix has been developed by Chen et al. [8].
They demonstrated that the cell volumetric response was influenced by several key factors, including the cell shape, the loading type, and the matrix Poisson ratio. The model predicts a range of testable behaviors of cells in a 3D matrix, including the development of cell alignment under mechanical stretching.

The proteins by which cells adhere to their microenvironments are critical players in mechanotransduction. Cell adhesion is thus a crucial factor in determining how cells sense and respond to their surrounding mechanical microenvironment, and also in how they maintain and modulate tissue integrity. A diffusive-stochastic-viscoelastic model has been developed by He et al. [9] to study adhesion of cells to viscoelastic solids via stochastically formed molecular bonds. Their results revealed that the adhesion lifetime and strength are determined by a competition between time scales of molecular diffusion, reaction kinetics, and viscoelastic deformation of the matrix. Because these factors are difficult to observe directly, testing models of this character requires predictions of secondary phenomena. In this case, an optimal molecular diffusion coefficient was identified, which was compared, favorably, against experimentally observed characteristic time constants for receptor diffusion on cell surfaces.

Cells typically use these adhesion protein dynamics to feel their mechanical microenvironment. These dynamics typically result in the application of fluctuating forces to the proteins surrounding a cell. However, the mechanisms underlying how cells apply fluctuating forces to sense matrix rigidity remain elusive. A generic filament-crosslinker modeling system for stress fibers was developed by Wei et al. [10] and used to investigate the force generation of proteins with biased binding and bond-breaking kinetics. Their results reveal that the fluctuating force profile is essentially due to force-induced structural instability during stress fiber assembly. This observation provides important insight into the process of cell durotaxis. Results suggest that cells utilize or circumvent such forces via biased kinetics. Once validated directly, the framework holds potential to understand how fluctuations underlie a broad range of mechanobiological processes.

Another feature of the ways that cells probe their microenvironment is the use of filopodia filled with parallel actin bundles. These have been modeled by Wang and Qian [11], who employ a coarse-grained molecular dynamics model to investigate how cross-linked actin bundles resist externally imposed compression. The bending stiffness of actin bundles is found to depend on the bundle size. The bending and buckling behaviors of the actin bundles are heavily influenced by the degree of coupling between actin filaments. These findings could be exploited to understand and eventually control cell morphology and migration through regulating buckling mechanics of filopodial protrusion. 
Applications of mechanobiology similarly require modeling. A canonical example is uptake of nanoparticles by cells, which is a key factor in modern, targeted drug delivery. From a range of mechanics solutions, the stiffness of cells is expected to play a role in the rate of nanoparticle endocytosis. Because stiffness varies among cell types and may change in pathological conditions such as cancer, and because mobility in the extracellular or interstitial matrix is affected by cell mechanics and inflammation, the mechanics of nanoparticles must be tuned carefully. The example in this special issue comes from Tian et al. [12], who investigate the diffusion of rigidity-tuned nanoparticles (e.g., drug nanocarriers) in biological hydrogels using coarse-grained molecular simulations. Their results show that the rigidity of nanoparticles influences their deformation, which dominates the diffusivity of nanoparticles in biological hydrogels. More broadly, their work highlights the central role of microenvironmental mechanical modeling in the range of diagnostic and treatment possibilities that the field of mechanobiology is opening.

\section{Perspectives}

The field of mechanobiology is beginning to provide insight into the pathogenesis and potential treatment of a range of diseases. A common theme that has arisen out of this field, and that is reflected throughout this special issue, is that the microenvironment of a cell-a cell's first and nearest contact to the world surrounding it-is critical to these outcomes. The diseases in which maladaptation to microenvironmental stimuli is a primary factor are amongst the most prevalent causes and contributors to mortality in the developed world. These include fibrosis and hypertension. Additionally, the metastasis of cancer is a fundamental mechanics problem, in that it necessarily involves migration out of a cell's natural microenvironment. The diseases of mechanics associated with mechanobiology represent challenges that face all of us as a global society. These challenges will be best overcome by continuing to work together as a global community, and we hope that this thematic issue will, like the conference that motivated it, continue to help bring together people with diverse scientific interests and backgrounds to address them.

Acknowledgements This major international undertaking was made possible through the coordinated administrative and financial support of a number of leading organizations and individuals across the globe. Support from the International Union of Theoretical and Applied Mechanics, the National Natural Science Foundation of China (Grants 11872298, 11602191, 11522219, and 11532009), the Projects of International (Regional) Cooperation and Exchanges of NSFC (11761161004), and the National 111 Program of China (B06024) is acknowledged, as well as support from the US NSF Science and Technology Center for Engineering MechanoBiology (CMMI 1548571). We thank the Bioinspired Engineering and Biomechanics Center at XJTU, the Mechanobiology Institute at the National University of Singapore, the University Alliance of the Silk Road, the McDonnell International Scholars Academy, and the US NIH/IMAG I $\mu$ BEAM working group for financial and administrative support. We thank all authors and reviewers for this special issue for their effort and time, and the staff of the editorial office of Acta Mechanica Sinica for their assistance.

\section{References}

1. Chen, J.W., Wang, N.: Tissue cell differentiation and multicellular evolution via cytoskeletal stiffening in mechanically stressed microenvironments. Acta Mech. Sin. (2018). https://doi. org/10.1007/s10409-018-0814-8

2. He, S.J., Li, X.M., Ji, B.H.: Mechanical force drives the polarization and orientation of cells: quantification of collective cell behaviors. https://doi.org/10.1007/s10409-019-00864-Z

3. You, Z.F., Zhou, L., Li, W.J., et al.: Mechanical microenvironments as the key cellular regulator in the liver. Acta Mech. Sin. (2019). https://doi.org/10.1007/s10409-019-00857-y

4. Xia, Y.T., Pfeifer, C.R., Discher, D.E.: Nuclear mechanics during constricted migration. Acta Mech. Sin. (2019). https://doi. org/10.1007/s10409-018-00836-9

5. Sun, Y.Y., Chen, G.B., Lv, Y.G.: Effects of hypoxia on the biological behavior of MSCs seeded in demineralized bone scaffolds with different stiffness. Acta Mech. Sin. (2019). https://doi. org/10.1007/s10409-019-00845-2

6. Wang, M., Liu, S.B., Li, F.: Imaging oxygen microenvironment in hydrogel microwell array. Acta Mech. Sin. (2019). https://doi. org/10.1007/s10409-018-0832-6

7. Gao, B., Jing, C., Ng, K.: Fabrication of three-dimensional islet models by geometry-controlled hanging drop method. Acta Mech. Sin. (2019). https://doi.org/10.1007/s10409-019-00856-Z

8. Chen, X., He, W., Liu, S.B.: Volumetric response of an ellipsoidal liquid inclusion: implications for cell mechanobiology. Acta Mech. Sin. (2019). https://doi.org/10.1007/s10409-019-00850-5

9. He, K.C., Li, L., Wang, J.Z.: Diffusive-stochastic-viscoelastic model on specific adhesion of viscoelastic solids via molecular bonds. Acta Mech. Sin. (2019). https://doi.org/10.1007/s1040 9-019-00848-Z

10. Wei, J., Chen, X.F., Chen, B.: Harnessing structural instability for cell durotaxis. Acta Mech. Sin. (2019). https://doi.org/10.1007/ s10409-019-00853-2

11. Wang, Y.Z., Qian, J.: Buckling of filamentous actin bundles in filopodial protrusions. Acta Mech. Sin. (2019). https://doi. org/10.1007/s10409-019-00838-1

12. Tian, F.L., Wang, H., Li, H.W.: Diffusion of rigidity-tuned nanoparticles in biological hydrogels-a molecular simulation study. Acta Mech. Sin. (2019). https://doi.org/10.1007/s10409-01900858-x

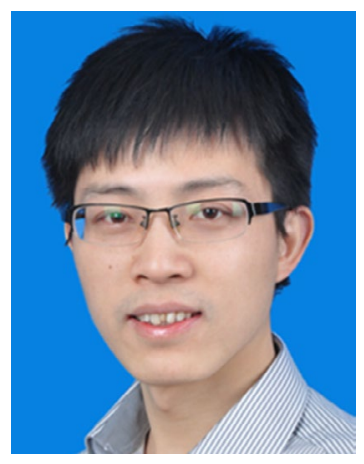

Guoyou Huang is an expert on advanced biomaterials and biotechnologies for control of the cell microenvironment. He serves on the Faculty of Life Sciences and Technology at Xi' an Jiaotong University, China. His training includes a B.S. in Physics in 2008 and a Ph.D. in Biomechanics in 2013 from Xi' an Jiaotong University, and training as a postdoctoral fellow at the Washington University School of Medicine in St. Louis, Missouri. He has extensive expertise with the 
synthesis, modeling, and micromanipulation of hydrogels for biomechanics, mechanobiology, and tissue engineering. His current research focuses on advanced biomaterials and technologies to engineer the cell microenvironment and to construct in vitro tissue models for fibrotic mechanotransduction, tissue engineering, and drug screening applications.

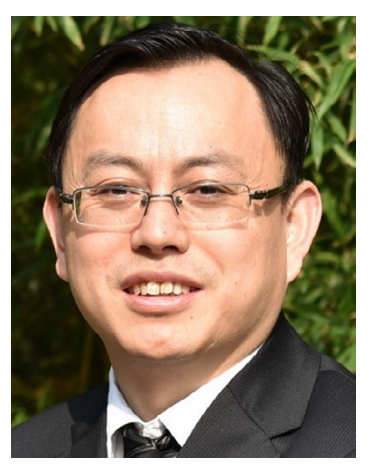

Feng $\mathrm{Xu}$ received his Ph.D. in Engineering from Cambridge University in 2008. From 2008 to 2011, he worked as a research fellow at Harvard Medical School and HarvardMIT Health Science and Technology (HST). He is cofounder and director of the Bioinspired Engineering and Biomechanics Center, the first interdisciplinary biomedical engineering center at $\mathrm{Xi}$ ' an Jiaotong University, China. He currently serves as Professor and Associate Dean at the School of Life Sciences and Technology, Xi'an Jiaotong University. His current research aims to advance human health through education and research that integrates engineering, science, biology, and medicine. He has received a number of awards for this work both nationally and internationally, including a track plenary lecture at the American Society of Mechanical Engineers annual IMECE meeting, State National Natural Science Awards, the NSFC Distinguished Young Scholar Award, and the Natural Science Award of the Ministry of Education of China.

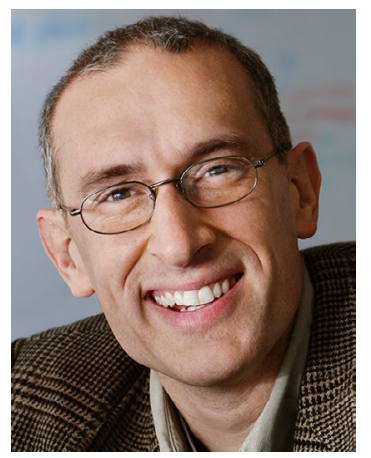

Guy M. Genin studies the mechanobiology of interfaces and adhesion. $\mathrm{He}$ is the Harold and Kathleen Faught Professor of Mechanical Engineering at Washington University in St. Louis, serving on the faculties of Mechanical Engineering and Materials Science, Biomedical Engineering, and Neurological Surgery. He is also Yangtze River Professor of Life Sciences and McDonnell International Scholars Academy Ambassador at Xi' an Jiaotong University in $\mathrm{Xi}$ 'an, China, and codirector of the Center for Engineer-

ing Mechanobiology, a joint NSF Science and Technology Center between Penn, Washington University, with several satellite sites. Prof. Genin serves as chief engineer for Washington University's Center for
Innovation in Neuroscience and Technology and is active in several start-ups. He co-chairs the NIH/Interagency Modeling and Analysis Group's working group on integrated multiscale biomechanics experiment and modeling. Prof. Genin's training includes B.S.C.E. and M.S. degrees from Case Western Reserve University, S.M. and Ph.D. degrees in solid mechanics from Harvard, and postdoctoral training at Cambridge and Brown. Prof. Genin is the recipient of a number of awards for engineering design, teaching, and research, including a Research Career Award from the NIH, the ASME Skalak Medal, and the Changjiang Scholar Award from the Chinese Ministry of Education. $\mathrm{He}$ is a fellow of the ASME and AIMBE.

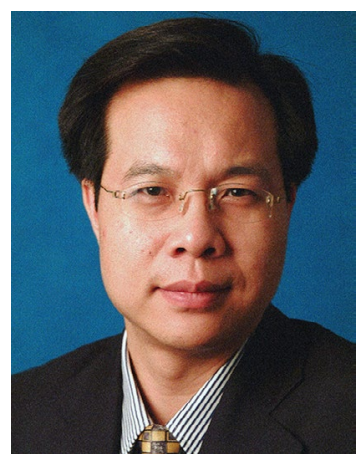

Tian Jian Lu received his Ph.D. in Engineering Sciences from Harvard University in 1995. Before taking up his current post at Nanjing University of Aeronautics and Astronautics, he was Professor and Vice President of Xi' an Jiaotong University (2004-2018) and Lecturer, Reader, and Professor of Materials Engineering at Cambridge University (1996-2006). He was also Fellow and Director of Studies of Queens' College, Cambridge University. He is the Founding Director of MOE Key Laboratory for Multifunctional Materials and Structures (LMMS). He uses theoretical, experimental, and numerical approaches to investigate key research frontiers in engineering sciences, addressing challenges in mechanics of materials, noise and vibration, heat transfer, and biomechanics. Between 2005 and 2016, he was the Chief Scientist for the National Basic Research Program (973 Project) of China. He is the recipient of many prestigious awards, including the National Natural Science Award of China. He is the Editor-in-Chief of Acta Mechanica Sinica (AMS), and serves as the Associate Editor or member of the Editorial Board for more than 10 professional journals. From 2010 to 2014, he served as the Vice President of the Chinese Society of Theoretical and Applied Mechanics (CSTAM). At present, representing China, he holds several important positions in the International Union of Theoretical and Applied Mechanics (IUTAM). He is a fellow of the AIMBE. 